\title{
Soft Architectural Assemblies: Soft Kinetics
}

\author{
VERA PARLAC
}

University of Calgary

The project presented in this paper explores integration of custom-made soft robotic muscles into a component-based surface. This project is part of a broader research agenda focused on new material behaviors and their capacity to produce adaptive and dynamic material systems. The paper discusses use of a pneumatic system as a form of materialbased and pneumatic actuation and presents ongoing research into the capacity of integrated pneu structures to generate kinetic movement within a component-based assembly to produce a responsive and 'programmable' architectural skin. This is a prototype-based exploration that demonstrates different kinds of movement achieved by different silicone muscle types and proposes a light modular construct, its components, and patterns of aggregation that work in unison with the silicone muscles to produce a dynamic architectural skin. The project is informed by a history of pneumatic structures, the technology of soft robotics, and a kit-of-parts design strategy.

\section{INTRODUCTION}

Pneu is a primary form of living nature, an effective structural system as well as an instrument of form giving. ${ }^{1}$ Every cell is a pneu structure. ${ }^{2}$ An elastic membrane that delineates pneu structures responds dynamically to the change of pressure by changing its mass. The change in pressure/mass can cause considerable physical transformation of the structure. This transformation is what enables a pneu structure to produce a kinetic effect in its own structure and also in structures attached to it. In engineering, rigid materials are employed to fabricate precise and predictable dynamic systems, but natural systems often exceed this performance with soft and flexible bodies. ${ }^{3}$

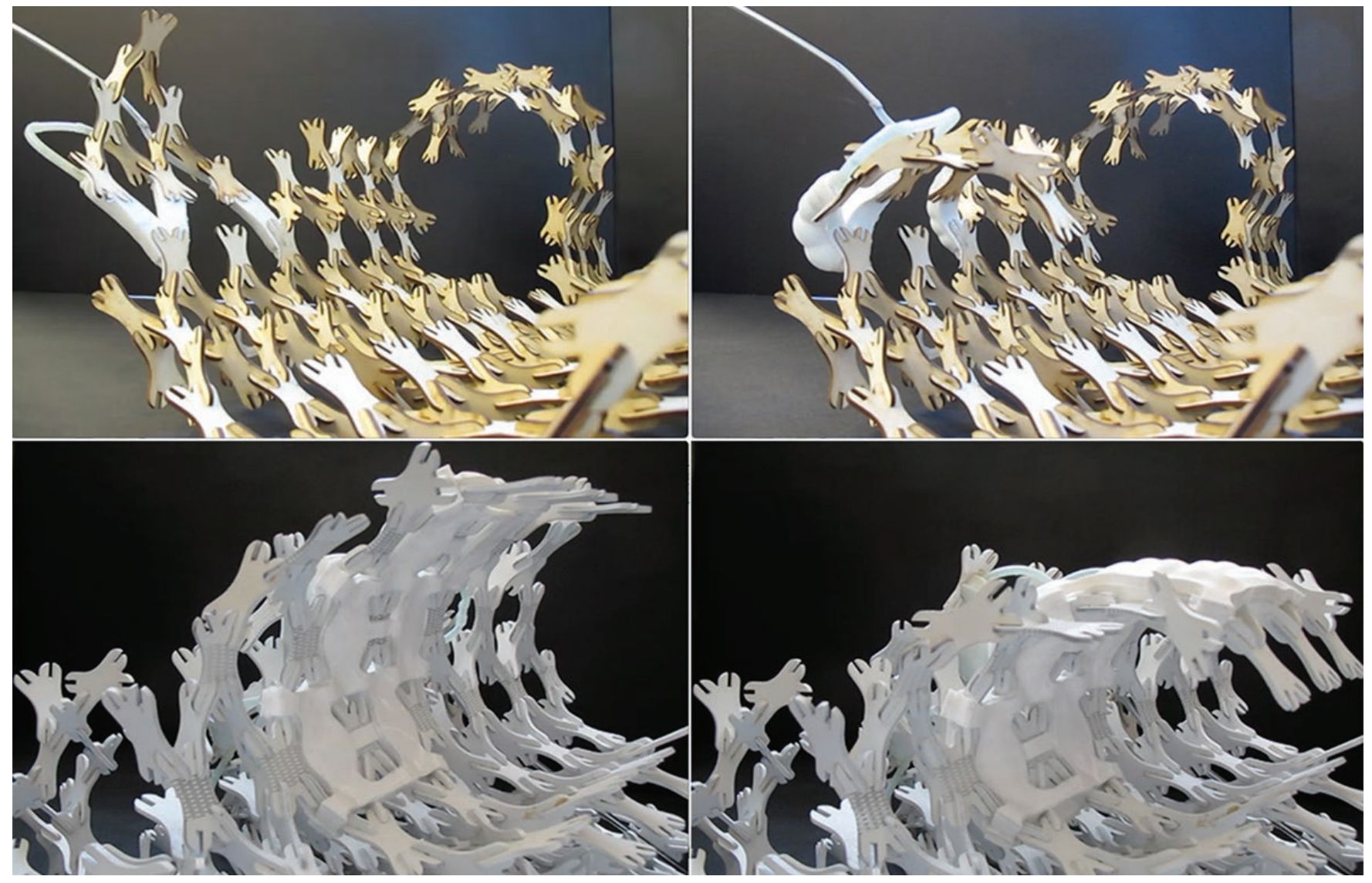

Figure 1. Active pneumatic muscles moving part of the structure, Copyright Vera Parlac. 
In soft robotics, the pneu-like capacity is used to design robots that move by manipulating the inflation and deflation patterns or handle fragile objects. Their bodies are capable of large-scale deformation and high level of compliance. ${ }^{4}$ Some of these robots are able to move around obstacles or squeeze under them. Research by Harvard's Biodesign Lab and soft robot fabrication techniques described by Andrew D. Marchese et al. ${ }^{5}$ provided a starting point for the initial studies of pneu elements used in this project. Other relevant studies are related to the nature of the soft actuator's movement and their motion patterns, ${ }^{6}$ and the complexity of this movement ${ }^{7}$. The capacity of soft robotic components to affect larger structures in which they are incorporated is of key importance for this project.

The project presented here attempts to address two challenges in designing dynamic and adaptive surfaces: actuation system and surface tectonics. In doing so it explores a capacity of pneu structures to induce kinetic movement in structures, and articulates a component based structure that facilitates movement. The project's main premise is that integration of elastic inflatable pneumatic components into a componentbased structure will influence the structure dynamically and support a variety of movements. The project is informed by a history of pneumatic structures, the technology of soft robotics, and a kit-of-parts design strategy (figure 1).

\section{BACKGROUND}

Inflatable or pneumatic structures have been used in architecture primarily for their lightness in relation to the structural span. Between 1940s and 1970s these structures underwent significant evolution. One of the first fully inflatable structures was a radome developed by Walter Bird in late 1940s. The exploration of air-supported structures quickly grew beyond their use as shelters for equipment or supplies; in 1960 s and 1970s they were used in a variety of experimental works in architecture. For example, the Fuji Group Pavilion, designed by Yutaka Murata for the 1970 World Expo in Osaka, was built using a series of attached air-inflated vinyl tubes that were bound together to form a larger structure, with the necessary air pressure maintained by the constant supply of air flowing through the tubes. The shift from a single inflated volume (radome) to the aggregation of inflated tubes presented an innovative moment in building large span pressurized structures. Besides their inherent self-supporting structural capacity, inflatable structures offered a potential to design soft and transformable spaces with new formal (and dynamic) qualities. Experiments by Coop Himmelb(I)au and Haus-Rucker-Co created structures at the scale of human body utilizing their soft and temporal qualities. A new kind of architectural spaces emerged that operated as an extension of the immediate body space. Coop Himmelb(I)au's Villa Rosa, for example, even allowed for a change of volume by inflation. These inflatable, mobile, and temporary structures were acknowledging transformational potential of the inflatable form. They brought into architecture the notions of a dynamic, changeable and soft space with boundaries no longer defined by rigid material enclosures. Today researchers are experimenting with even smaller scale inflatables that could be integrated into architectural surfaces and components i.e. into tectonics of a material system. Current experiments with elastic inflatable elements influenced by soft robotics are suggesting new trajectories in exploring dynamic spatial boundaries.

Soft robotics is concerned with the development of actuators made from "soft" materials. Materials such as shape memory alloy, electroactive polymers or silicone elastomers are used for these purposes. From the design point of view, soft actuators produce "soft" deformations and life-like movements, offering a large degree of freedom when conceptualizing adaptive building systems. ${ }^{8}$

Several experimental research projects point to a new potential that these structures might have in designing responsive and adaptive architectural surfaces and structures. The PneumaKnit by Sean Ahlquist, McGee and Sharmin ${ }^{9}$ explored motion and geometric articulation of inflatable components using knitted constraints. Instead of relying on the shape of the actuator to produce specific motion, knitted constraints regulate the expansion of the actuator and therefore its motion. The emphasis is on the material structure of the knitted constraint, which, through a density of its weaves, produces the surface transformation. This work is concerned not only with the actuator itself but also with the integrated system where an actuator and its constricting surface work in unison to produce the desired effect. The material integration between the inflatable and knitted elements is a step forward in rethinking the assembly of a material system in which constituent parts are dynamic and perform synergistically. On the other hand, in the Pneuma-Technics ${ }^{10}$, the focus is on the shape of the actuator itself. The difference in its size and shape and in geometry of its internal channels produces variations in its elasticity and therefore its directional deformation. This project offers a surface made of soft pneumatic components that can respond and adapt to modulate passage of light, air or view. The result is a soft panelized surface that can open and close through its inflation and deflation patterns. This is similar to Park's Modular Pneu-Façade System, imagined as "a dynamic pneumatic interface which can be used in building applications including responsive façade, ceiling, floor and interior screen, etc. ${ }^{\prime 11}$ The strong analogy of this system to the cardiovascular system in a body and other biological systems like muscular-hydrostats brings forward architects' fascination with "living" and sentient systems and the desire to tap into their potential for adaptation. The surface utilizes capacitive sensors and conductive gel, which make it sensitive and conductive to human touch. This layer of soft inflatable elements can be integrated into building skins to make them transformable and active. 

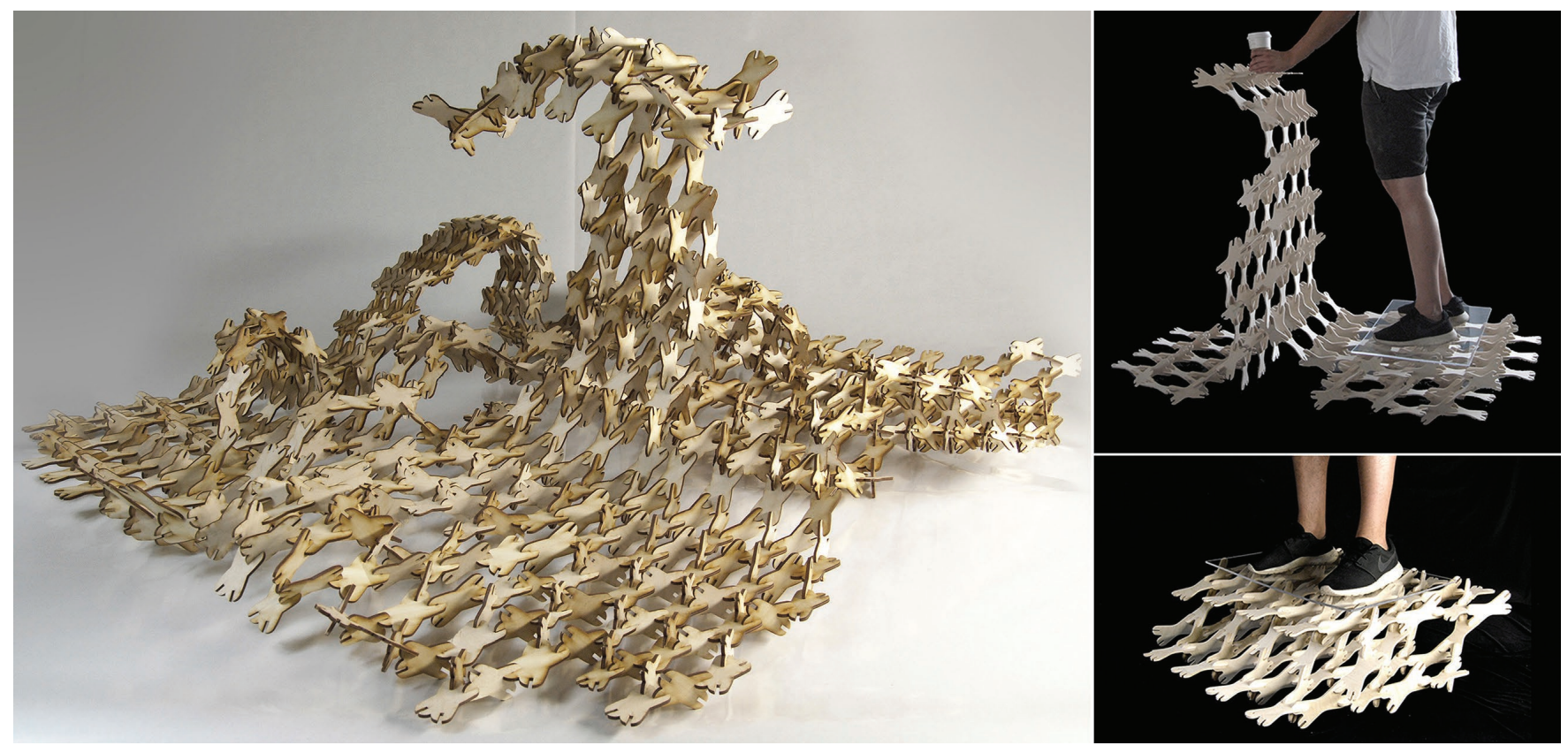

Figure 2. Rigid and pliant configuration, Copyright Vera Parlac.

The Soft Kinetics project, described in this paper, explores integration of soft and hard elements (or layers) to produce a dynamic material system that is self-supporting, pliable and kinetic depending on the configuration of modular components and the position and concentration of pneumatic elements. As such, it can be developed as a stand-alone building skin system that is at the same time a 'programable' surface. The goal of the project is to engage a capacity of these active structures (inflatable muscles) as ingredients of space making by exploiting their transformational capacity.

\section{METHODS}

The project presented here brings together two strategies for designing adaptive architectural skins. One is concerned with the combinatorial variability of a light structure built by aggregating small self-similar components. The other one focuses on the integration and distribution of pneumatic muscles within an aggregated structure. The proposed system reflects a deep interest in the development of a component-based material system whose properties range from rigid/stable (self-supporting) to pliable/active (dynamic). To regulate rigidity of the proposed material system particular attention was placed on emerging morphology of the proposed system that can produce modulated rigidity. This morphology is constructed by integrating self-similar components and animated by pneumatic muscles producing a 'programable' surface that can open, close or alter its basic form.

\section{LIGHT MODULAR STRUCTURE}

The light modular structure is built using self-similar elements with a non-orthogonal alignment. It is aggregated through slot-friction connections and can be organized in a number of different configurations. The configuration of the construct is governed by the requirements for stability (self-support) and kinetics, and both of these criteria are equally important to support dynamic transformations. Stability is achieved in two ways: by interlocking the components through simple slotfriction connections and by the patterns of aggregation. The kinetic behavior is enabled by a system of pneumatic muscles, their full integration with the patterns of aggregation, and the capacity of the modular structure to allow for disruptions in pattern continuity without compromising the construct's stability. The redundancy of connections and elements provides structural resiliency.

By utilizing a kit-of-parts design strategy the structure can be built in a variety of configurations and adapted to a variety of spaces. Due to the self-similar unit shape and the standardized connection between the units, there is a great combinatorial potential for a structure assembly. On a more local scale, however, the combinatorial potential is driven by the assembly pattern that is contingent on the shape of the unit and the angle of its connections. Following the assembly pattern, two main configuration trajectories emerged: rigid (self-supporting) and pliant (flexible) (figure 2).

Individual components could form any number of permutations, but discrete assemblies, used to govern the form of a larger construct, were generated to support change in functionality, directionality and form. These discrete assemblies were then combined into larger formations and their tectonic and spatial capacities were examined. However, the system itself remains open and able to adjust to a variety of spatial/contextual conditions as well as to support part replacement. In this way, recalibration of the construct can be maintained since its parts could be reconfigured in a variety of ways (figure 3 ). 


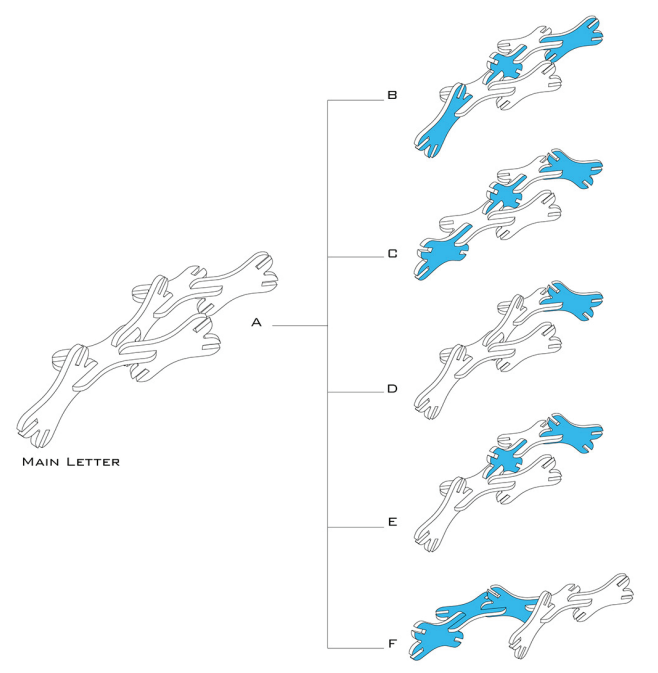

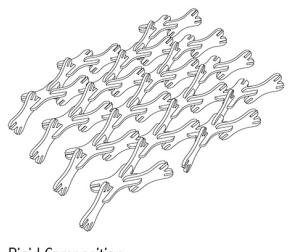

Rigid Composition

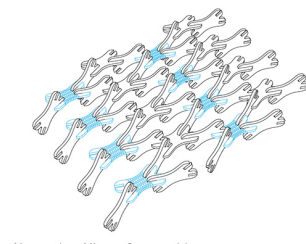

Alternating Hinge Composition

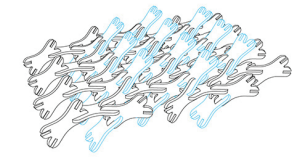

Double joint composition | Rigid Structure

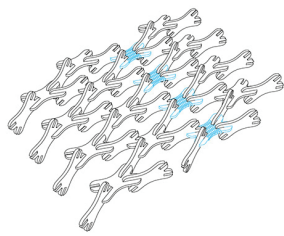

Hinge Composition

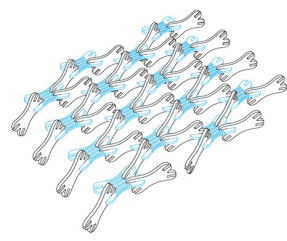

Pliant Field Composition

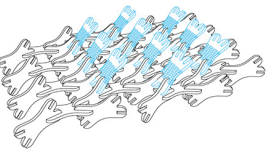

Pliant Structure

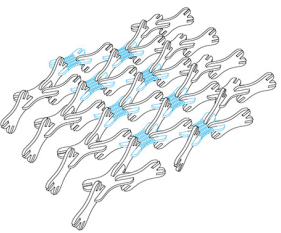

Double hinge Composition

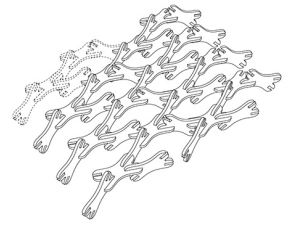

Letter D Rigid Composition

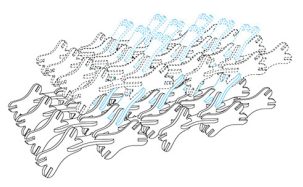

Letter A Rigid Composition

Figure 3. Combinatorial Potential, Copyright Vera Parlac.

The component shape was chosen for its capacity to produce a significant number of different combinations while maintaining the pattern that generates rigid and pliant versions. These configurations were then modeled digitally and tested physically for their behavior. The tests resulted in a design of a new bendable component that would be positioned adjacent to pneumatic muscles to facilitated bending of the regions surrounding the muscles. Ultimately, the modular structure can negotiate a change in direction (straight, angled, curved), change in thickness by smoothly transitioning from single to multiple layers (from a surface to a three-dimensional construct) and change in structural capacity (from self-supporting to bendable).

\section{PNEUMATIC MUSCLES}

The soft body of the Soft Kinetics project is imagined as a continuous and interrelated network of pneumatic muscles integrated into an assembly pattern of the modular structure. The soft body consists of clusters of interconnected soft inflatable pneumatic muscles. They are linked by silicone tubes that allow passage of air through a number of muscles, inflating and deflating them in a sequence. When inflated or deflated, these clusters move entire regions of close (figure 4).

The movement of pneumatic muscles depends on the flexibility of the elastic material and the volume of internal chambers and their geometry. ${ }^{12}$ Andrew Marchese et al (2015) list three soft robot morphologies differentiated by their internal channel structure: ribbed, cylindrical, and pleated. ${ }^{13}$ The soft body (actuation system) of the Soft Kinetics project is developed using a ribbed morphology but its internal channels are produced using two different techniques: the lost wax casting and a combination of the lamination casting and the soft lithography fabrication method. As a result, two types of muscles are produced: the central channel muscles $(S, B$, and V) and the distributed channel muscle (M) (figure 5). The fabrication technique is important in achieving consistent properties of the muscles as they get reproduced; it plays an important role in achieving consistent elasticity and inner channel geometry.

The behavior of designed pneumatic muscles was explored through prototyping and iterative design and their performance observed as they were integrated into a modular structure. The central channel muscle produced using the lost wax technique was resilient and durable (by not being cast in laminated fashion). It achieved significant bending compared to the distributed channel muscle. In general the lost wax technique allowed for a great variety of cavity forms since the muscle was made as a solid body and was designed that way, not in layers. The muscles underwent several modifications to achieve maximum bending after inflation.

The muscles were conceived as modular elements of the structure and as such could be integrated into the structure interchangeably; two muscles ( $S$ and V) were designed to integrate into the assembly grid pattern while the other two ( $B$ and $M$ ) nest within the voids of the grid. The central channel type muscles act as linear actuators while the distributed channel muscle acts as a folding hinge (figure 5). 


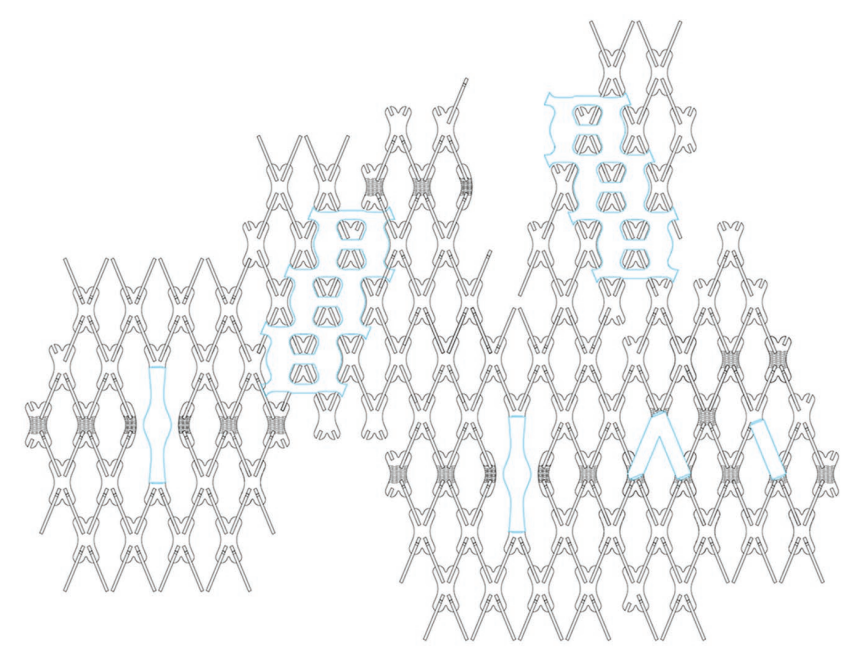

Figure 4. Assembly pattern and surface deformation, Copyright Vera Parlac.

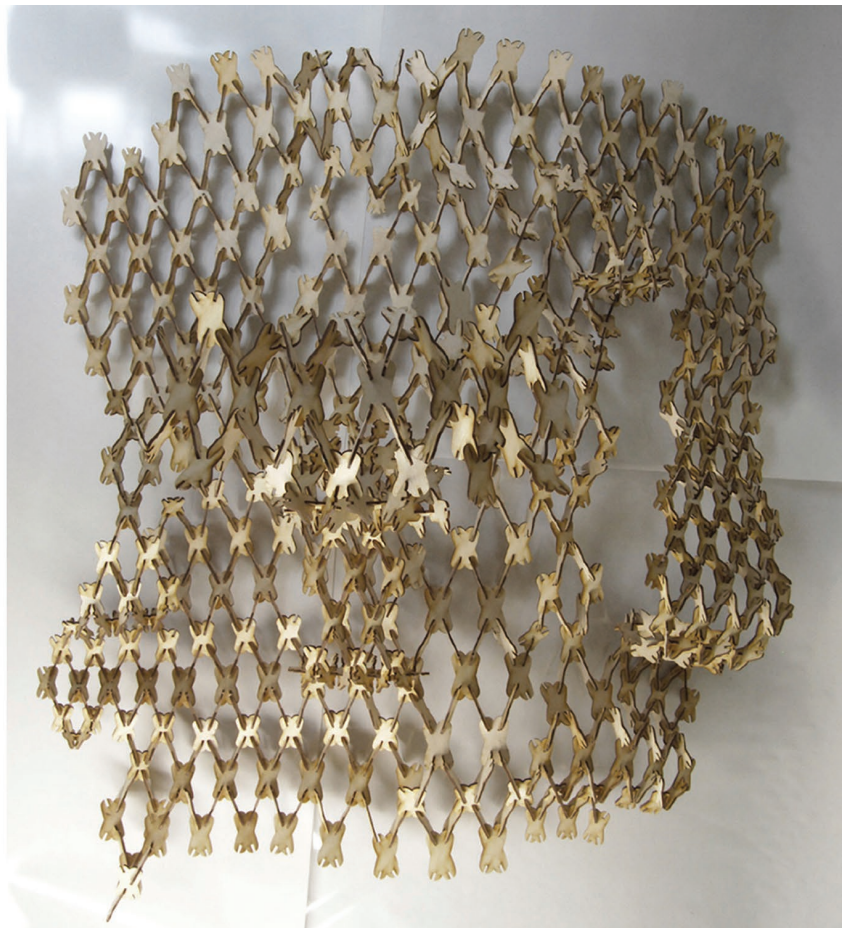

The muscle morphology and geometry were designed to balance the wall thickness and the volume of air channels. These two different types of muscles required different approaches to mold production. The central channel muscle molds were CNC milled, while the distributed channel mold was produced by layering laser-cut acrylic material. Both types of muscles were made from silicon rubber. To direct their motion, one side was fabric-reinforced. Soft elastomer pneumatic muscles are capable of continuous deformation but the challenge is to isolate a particular bending movement within its length. ${ }^{14}$ For that reason, the pneumatic muscles used in this project were made short and compliant with the grid pattern of the modular structure (figure 5); their length and cluster organization, however, will be further explored in the next phase of the project, to produce asymmetrical shifts within the fabric of the modular structure.

\section{INTEGRATION AND PROTOTYPES}

The "soft" body of pneumatic muscles is integrated with the "hard" body of the light modular structure and can be distributed in a variety of ways to produce a specific kinetic result. The integration is achieved by embedding the modular component of the structure into the central channel muscles and by inserting the components into openings in the distributed channel muscles (figure 5).

The central channel muscles were integrated by a slip joint into the hard body of the structure just like any other modular component of the system. Therefore, the soft pneumatic muscles can be positioned to displace the "hard" parts of the modular structure, working as an active connective tissue, while the overall assembly pattern was maintained. This strategy allows muscles to be asymmetrically distributed throughout the structure, concentrated in some areas, or placed sporadically in others. This is seen as a very promising direction that will be further explored in the next phase of the project. For example, larger segments of the modular structure could be dynamically altered to open and close apertures of varying sizes and shapes (figure 5).

Several small prototypes were constructed to test various ways and combinations of muscle integration. It is in the prototypes that the clustering of muscles was explored. The clusters, consisting of three to five pneumatic muscles, were inflated in sequence. Solenoid valves controlled the inflation and deflation pattern (supply and exhaust) and their work was regulated through an Arduino microcontroller. The rate and duration of valve opening and closing was set to allow all linked muscles to inflate in a sequence; the pressure was controlled through a sensor to prevent over-inflation and damage to the muscles. Muscles linked in a cluster worked as a group, affecting dynamically a designated region of the structure. The work of the "soft" body that opens and closes the apertures can be controlled through proximity sensors to reveal a clear view out or in, and can be regulated by light sensors to serve as a functioning shading device. 

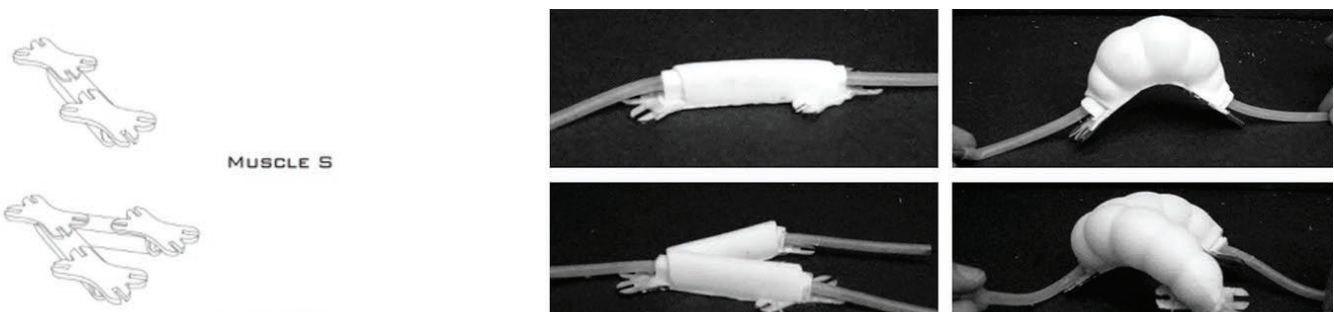

Muscle $V$
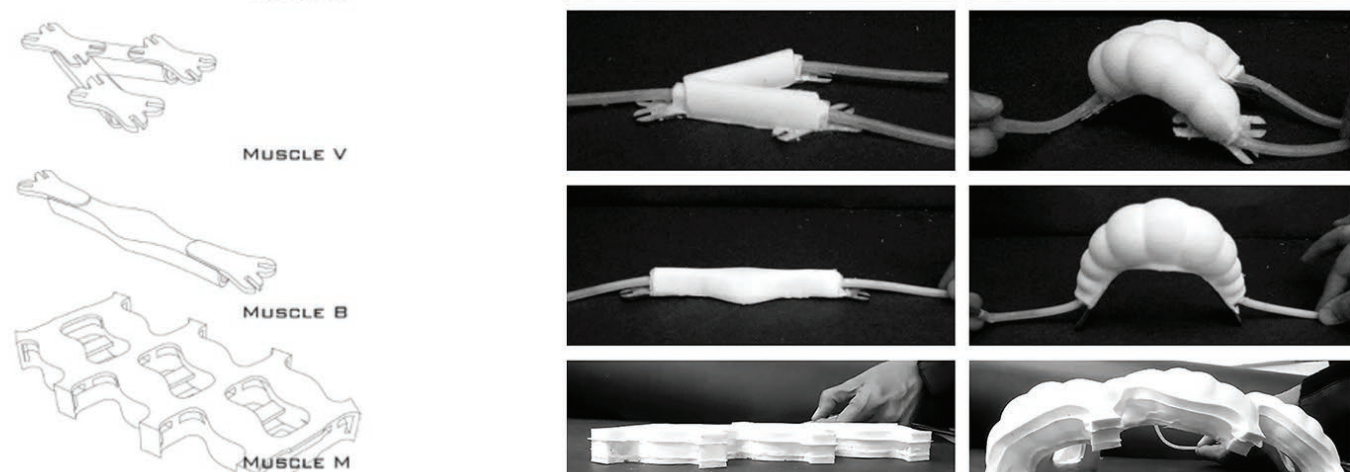

MUSCLE M
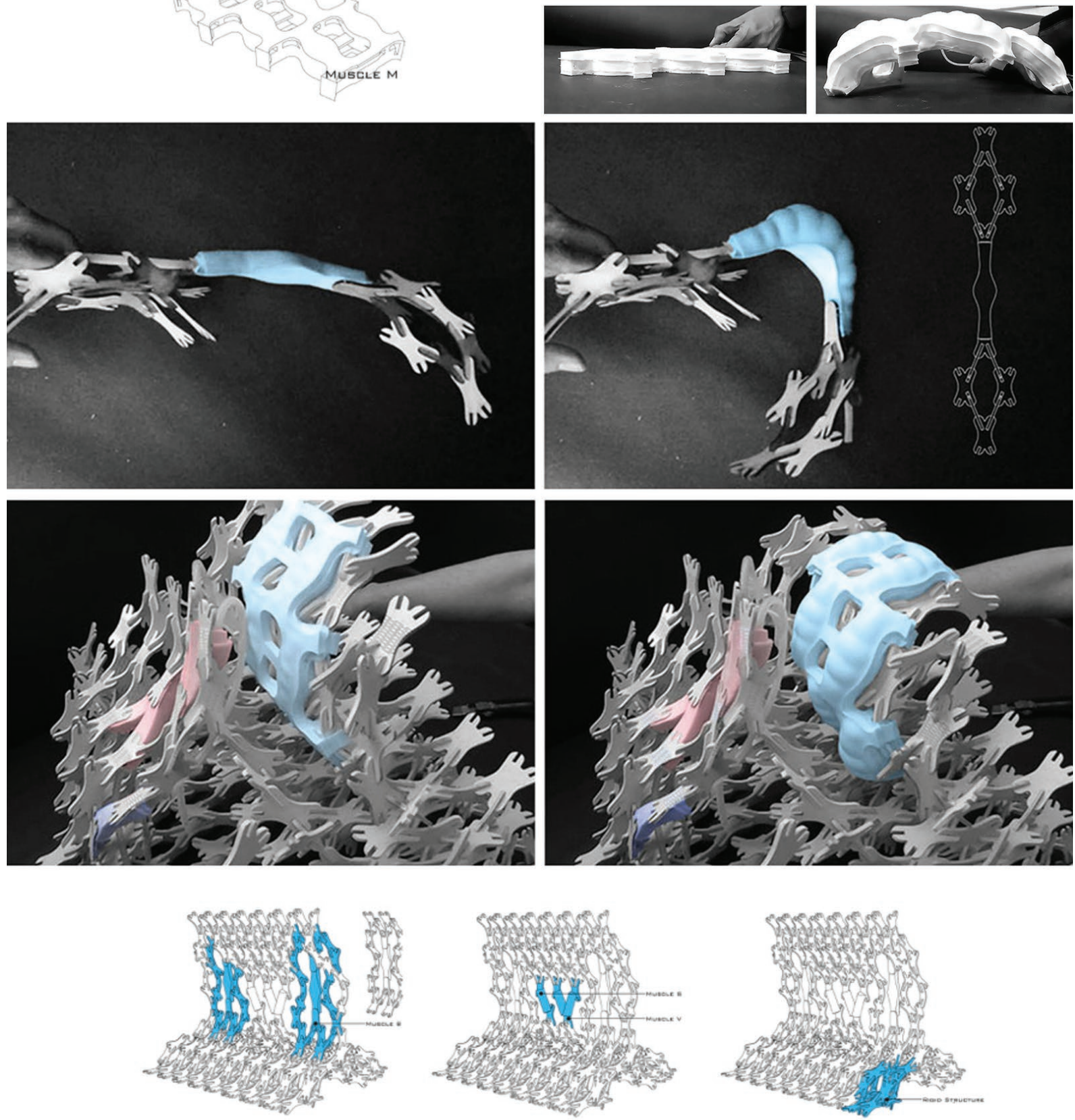

Figure 5. Muscle types and their performance; testing the connection and movement of the muscle; dormant and active muscles; illustration of muscle integration, Copyright Vera Parlac. 


\section{RESULTS AND DISCUSSION}

The research so far shows a promising way of integrating an active pneumatic layer within a light modular structure. An important aspect of this work is the transitioning between fixed and dynamic regions of an aggregated structure without any additional components to facilitate that transition and compensate for motion. This is achieved by using self-similar components and by designing some of those as bendable. Other essential features are the slot-friction connections, consistent aggregation patterns, and modular pneumatic components that integrate seamlessly into the designed patterns. The transitions between dynamic and static regions are achieved through carefully designed (and tested) component distribution patterns and varying densities of pneumatic muscle components within the aggregated construct. Parts of the resulting structure are self-supporting, providing structural stability, while other parts permit different levels of movement without compromising the structural integrity. This variation in performance is achieved by a variable density of the assembly pattern and its extensive combinatorial capacity.

The Soft Kinetics project introduces small-scale pneumatic components and weaves together soft, hard and pliable elements to create a material system that can behave dynamically in a variety of ways and transition seamlessly between them. The design of pneumatic muscles builds on projects such as the Pneuma-Technics ${ }^{15}$ and the Modular Pneu-Façade System, ${ }^{16}$ but strives to seamlessly integrate soft and hard layers into a composite system using the same aggregation patterns. The key feature of the project is an actuating system that is embedded through the geometry of its components into the overall structural pattern of the construct while simultaneously making the regions of that construct dynamic. This is achieved through the modularity and the use of slot friction connection for all components in the assembly, including the pneumatic muscles. In nature functionality and materiality of the material systems are blended and integrated. This project attempts to integrate those capacities within an architectural assembly, by integrating functionality and materiality of soft/dynamic and hard/structural layers to produce dynamic architectural assemblies.

\section{CONCLUSION AND FUTURE WORK}

The main motivation for the development of this system of soft actuation is to produce a uniform material system that can be built in a variety of configurations and reduce a presence of complex mechanical systems in dynamic architectural assemblies (such as dynamic building facades). The primary advantage of this mode of actuation is its lightness, as well as the possible degree of movement control that it offers.

The weight of the structure is an important factor; therefore more research is required to define the "blended" materiality of the modular structure as well as the durability and weight of the embedded pneumatic components. Composite structure is currently made of plywood but the use of aluminum and plastic will be explored in future iterations.

The integration of actuators within the aggregation pattern of the structure allows for movement of its regions. In the current study, small clusters of actuators and their behavior were examined. The distribution and density of the actuators need further study to fully understand the potential of this system to move larger regions of a structure. New computational tools for simulation of complex non-elastic behavior will be employed so that alternate designs could be quickly tested. Future research will also focus on the design of alternate aggregation patterns to further experiment with the range and amplitude of motions.

In his seminal article on Resilience and Stability of Ecological Systems C. S. Holling point out different ways we see the behavior of a system. Engineered systems or devices that perform specific tasks under predicable external conditions have their performance immediately adjusted if the variation in performance is observed. They are concerned with constancy of performance and lean towards stability. Natural systems that are constantly confronted with unpredictable external changes are less concerned with constancy and more with persistence of the relationships. ${ }^{17}$ An equilibrium-centered view of a system is static and doesn't offer flexibility necessary for systems with transient behavior. Project presented here is an exploration into how to incorporate variability in performance (from stable/structural to dynamic) and how those variable conditions integrate and interface with each other.

When designing active and adaptive artificial environments, whether they are intelligent facades or built environments that interface with natural ecologies, we want to establish a flow of information and energy. The adaptive synthetic built environment should behave similarly to natural systems and therefore we might be less interested in stability as an on or off condition and more in the zone of stability, its gradient and ability to perform under the constant change.

\section{ENDNOTES}

1 Frei Otto, "Pneus in Living Nature," in IL35 Pneu and Bone (Stuttgart, Germany: Institute for Lightweight Structures (IL), 1995), 56-63.

2 Johann-Gerhard Helmcke, "The Biological Pneu," in IL9 Pneus in Nature and Engineering (Stuttgart, Germany: Institute for Lightweight Structures (IL), 1977), 138-150.

3 Daniela Rus and Michael T Tolley, "Design, Fabrication and Control of Soft Robots," Nature 521 (May 2015): 467-475.

4 Andrew D. Marchese, Robert K. Katzschmann, and Daniela Rus, "A Recipe for Soft Fluidic Elastomer Robots," Soft Robotics 2, no. 1 (2015): 7-25.

5 Marchese, Katzschmann, and Rus, 7-25.

6 Joshua Bishop-Moser, Joshua Girish Krishnan, Charles Kim, and Sridhar Kota, "Design of Soft Robotic Actuators Using Fluid-Filled Fiber-Reinforced Elastomeric Enclosures in Parallel Combinations," in 2012 IEEE/RSJ International Conference on Intelligent Robots and Systems (October 2012): 4264-4269.

7 Fionnuala Connolly, Conor J. Walsh, and Katia Bertoldi, "Using Analytical Modeling to Design Customized Fiber-Reinforced Soft Actuators," in Society of Engineering Science 53rd Annual Technical Meeting (October 2016). 
8 Dino Rossi, Zoltán Nagy, and Arno Schlueter, "Soft Robotics for Architects: Integrating Soft Robotics Education in an Architectural Context," Soft Robotics 1 no. 2 (2014): 147-153

9 Sean Ahlquist, Wes McGee, and Shahida Sharmin, "PneumaKnit: Actuated Architectures Through Wale- and Course-Wise Tubular Knit-Constrained Pneumatic Systems" in ACADIA 2017: Disciplines and Disruptions, Proceedings of the 37th Annual Conference of the Association for Computer Aided Design in Architecture, ed., T. Nagakura (Cambridge, MA: ACADIA, 2017), 38-51.

10 Daniel Fougere, Ryan Goold, and Kathy Velikov, "Pneuma-Technics: Methods for Soft Adaptive Environments" in ACADIA 2015: Computational Ecologies: Design in the Anthropocene, Proceedings of the 35th Annual Conference of the Association for Computer Aided Design in Architecture, eds., L. Combs and C. Perry (Cincinnati: ACADIA, 2015), 274-283.

11 Daekwon Park and Martin Bechthold, "Designing Biologically-Inspired Smart Building Systems: Processes and Guidelines," International Journal of Architectural Computing 11, no. 4 (2013): 437-464.

12 Bishop-Moser, Krishnan, Kim, and Kota, "Design of Soft Robotic Actuators Using Fluid-Filled Fiber-Reinforced Elastomeric Enclosures in Parallel Combinations," 4264-4269

13 Marchese, Katzschmann, and Rus, "A Recipe for Soft Fluidic Elastomer Robots," 7-25

14 Ahlquist, McGee, and Sharmin, "PneumaKnit: Actuated Architectures Through Wale- and Course-Wise Tubular Knit-Constrained Pneumatic Systems," 38-51.

15 Fougere, Goold, and Velikov, "Pneuma-Technics: Methods for Soft Adaptive Environments," 274-283.

16 Park and Bechthold, "Designing Biologically-Inspired Smart Building Systems: Processes and Guidelines," 437-464

17 C. S. Holling, "Resilience And Stability Of Ecological Systems," in Annual Review of Ecology and Systematics, Annual Reviews 4 (1973): 1-23. 